\title{
How Safety Communication Can Support Safety Management: The Case of Commercial Aviation
}

\author{
Michel Guérard
}

\begin{abstract}
Commercial aviation traffic has increased so dramatically over the past decades that virtually everyone can identify him/herself to a passenger or a passenger's relative. With the evolution of communication means and pace, every accident or incident induces an unprecedented amount of reactions and communication from many actors outside the aviation community. These newcomers on the safety communication scene challenge the historical safety management world and actors, traditionally limited to aviation professionals.
\end{abstract}

Keywords Safety management - Aviation - Safety communication

\section{Introduction}

Air travel has grown steadily since the end of the Second World War, doubling every 15 years. This industry is still expanding at roughly the same rate thanks to the emergence of new markets as well as to the development of new business models such as low-cost carriers.

Had the accident rates of the 60s and the 70s been kept at the same levels, the air transport safety situation would have become unacceptable to the travelling public and the development of the air travel industry would have unavoidably been severely impacted. The average fatal accident rate during the 60 s was 4.4 per million flights, compared with 0.15 per million flights in 2016. An American researcher named Curt Graeber published in 1995 a study in which he applied the then prevailing accident rates to traffic projections and concluded that unless something was done in terms of safety, the industry would have to cope with one serious accident every week by 2010 (Graeber 1995). This conclusion did not go unnoticed and strongly contributed to the promotion of safety enhancement initiatives within the air travel industry.

\footnotetext{
M. Guérard (凹)

Toulouse, France

e-mail: michel.guerard@airbus.com

(C) The Author(s) 2018

M. Bourrier and C. Bieder (eds.), Risk Communication for the Future, SpringerBriefs

in Safety Management, https://doi.org/10.1007/978-3-319-74098-0_9
} 
This reasoning was perfectly sound but this terrible prophecy never materialized, because in the meantime safety drastically improved. However, this frightening warning had been heard worldwide and positively contributed to the increased mobilization of the entire industry towards a safer air transport system. Graeber's study was not only about safety projections; it was also about communicating them to all stakeholders in a powerful and effective way.

With the spectacular development of commercial air transport around the world in the past decades, a growing number of people feel concerned by aircraft accidents. Air transport is an international activity by nature, and from an outsider's vantage point, nothing looks more like an airline, an airport or an aircraft than another airline, airport or aircraft. Almost everyone can identify him/herself to a passenger or a passenger's relative.

In addition, the accelerated development of information and communication means and channels makes each and every event, especially significant incidents or accidents known to almost everyone on the planet, and its interpretation by whoever communicates on it as well.

Over the years, safety-related topics have progressively been dealt with by new audiences, such as Internet sites, independent experts, lawyers, victims' associations, social media groups, pilot unions. The objectives were not the same and comments could sometimes be critical towards the air transport industry. This has opened new issues for air travel industry safety communications specialists, who found themselves facing higher levels of criticism than ever before.

In parallel, the overall image of the aviation industry has changed. Air transport began as a dream, even though only a few people were initially directly concerned. The early years of aviation triggered considerable enthusiasm among vast portions of the population. As it became a more banal way of moving around, the acceptability of accidents decreased and the identification of the public at large to air travellers increased, since more and more people could afford travelling by air.

The enthusiasm that initially surrounded air travel progressively faded and new environmental preoccupations arose. Aviation has been progressively associated with noise, pollution and global warming. Building a new airport or simply adding a runway to an existing one can be a major challenge in many countries. The difficulties encountered in Japan in the 70s concerning Narita airport and those concerning Heathrow's fifth terminal in the 80s or recently in France for the projected new Nantes airport illustrate this situation.

This changing environment is a challenge for the air travel industry in terms of communication. In other words, the perception framework of commercial air transport safety has evolved significantly.

If safety communication has always existed, its scope, actors and pace have changed, thus modifying its impact on safety management. It is therefore in the interest of this industry to better understand the ongoing changes in order to address them in the best possible way. 


\section{Safety Communication Among Industry Actors: A Historical Driver for Safety Enhancement}

Organizing activity in commercial air transport dates back to its earliest years, after World War II. Indeed, ICAO was created in 1944 and IATA followed in 1945. As for Authorities, they were already in place in the countries where air transport had begun. Most legacy carriers were created around that time and the then big manufacturers (Lockheed, Douglas, Boeing, De Havilland, etc.) were already producing airliners.

One can say that a solid frame was in place and ready to be used for safety matters as well. In fact, aviation actors realized fairly early that safety was not a matter of competition but rather a condition for the survival and the development of the industry as a whole. Indeed, whatever the area of the world, the aircraft operated and regulations are similar, as are air traffic control mechanisms. In other words, sharing information about safety has been identified as a crucial need for a long time.

The US National Transportation Safety Board, for example, has been keeping records of civil aircraft accidents since 1967. The National Aeronautics and Space administration administers a confidential and voluntary Aviation Safety reporting System since 1976.

However, with the evolution of technology and culture, the types of information gathered and shared as well as sharing practices evolved over time. It started with the lessons learned from accidents identified as an obvious source of information to contribute to improve safety. This sharing between operators did indeed help to identify major hazards, such as the metal fatigue phenomenon that was only understood after the De Havilland Comet fatal crashes in the 50s, and 'deep stalls' that were not known until the 1963 BAC 1-11 accident.

Experience feedback has always been and still is a vital flow, especially in aviation, because airliners are complex machines which are flying in increasingly large numbers. In addition, every new aircraft experiences its lot of early problems, but it will stay in service for 20 to 30 years; ageing problems will in turn feed this experience feedback. In other words, disseminating across the entire industry the lessons learned from adverse safety events has always been and will remain of prime importance to enhance safety.

As a matter of fact, in order to further improve safety, authorities have made it mandatory to report significant events, regulating in a sense one of the aspects of safety communication. In Europe, for example, occurrence reporting is defined in a document called AMC 20-8. However, the resourcing of aviation authorities is variable and this basic two-way flow of information is not always in place. It nonetheless forms the basis of a learning process that constitutes the foundation of industry-driven safety communication. If accident and serious incident investigations enable us to learn from what happened, they fortunately remain very rare events. All potential safety events do not end up in incidents or accidents.

The main stake for an industry growing at such a pace became to learn from other sources of information in order to try to further enhance safety. Therefore, voluntary reports from crews were encouraged by Authorities and within airlines in order to 
gather additional information on actual practices possibly inducing safety issues. This new form of safety communication involved setting up a specific framework. Indeed, voluntary reports were much more numerous in environments where they were not systematically punished when reporting mistakes, i.e. where 'just culture' principles (Havinga and Dekker 2014) translated into practice. A blame culture does still exist in certain parts of the world and deprives organizations and the industry as a whole of an opportunity to learn from experience. Safety communication is a powerful tool in terms of safety enhancement, but it must be fed.

With technological progress in terms of data recording and processing, another source of information was made available to further enhance safety: flight data analysis. A number of parameters are systematically recorded all along a flight and deviations from a safe envelope defined a priori are pointed out. Therefore, even in the absence of any incident or accident, safety-related information can be retrieved, processed and the outcome of the safety analysis disseminated. In a more recent approach, an auditor may also fly with a crew in order to attend the flight and evaluate the general performance of the pilots. ${ }^{1}$

This shows how far the air transport industry is ready to go in order to learn from day-to-day operations and to collect safety-related information. These various sources of information are potentially a very effective way of enhancing safety, but require a good safety communication system both within the operator and outside. In fact, practices in terms of communication to feed the analysis and disseminate the safety lessons learned may vary significantly around the world.

It is worth noting that most of these safety enhancement practices were put in place by numerous operators even before they became mandatory. In other words, safety communication is regulated between individual aviation actors and the Authorities. However, as mentioned earlier, the industry has soon realized that safety is a matter of survival of the activity as well. Therefore, additional safety communication practices developed with time on a voluntary basis.

In the US, a system was launched in October 2007, and an associated forum as well, for all aviation actors to share their safety information and perform joint analysis: Aviation Safety Information Analysis and Sharing (ASIAS). Volunteer airlines, airports and air traffic control centres get together on a regular basis, share their flight data analysis, incident reports and other sources of information to collectively learn lessons from operations. A similar approach is currently under study at European level.

Aircraft manufacturers also communicate about safety on a regular basis with airlines. It is done through technical bulletins, safety conferences and magazines. Similarly, airlines report operational occurrences to manufacturers on a voluntary basis. The number of events reported increases regularly, because airlines increasingly understand the benefits of reporting and communicating about their events. More generally, the awareness of all aviation actors of the benefits to learn from as much experience as possible is increasing. Indeed, even though each airline

\footnotetext{
${ }^{1}$ A mechanism known as Line Operations Safety Assessment, or LOSA.
} 
operates a limited number of aircraft, in a limited number of operational conditions, the overall experience accumulated by the industry is huge, since there is more than one aircraft taking off every second around the globe. However, as mentioned earlier, even if the safety communication system collects more and more data, with again some variations among actors, making sure that the appropriate safety information reaches the relevant targets is a different story.

Indeed, experience has shown that sometimes safety lessons were shared among safety managers but stayed with them, without reaching the operational people concerned.

This internal aspect of safety communication is now addressed by a recent regulatory requirement. Indeed, safety promotion within an organization is one of the four pillars of the Safety Management System (SMS) regulation. SMS is basically a new approach to safety which asks aviation actors to identify and evaluate their risks in order to mitigate them and therefore enhance safety. It is detailed in the International Civil Aviation Organization Annex 19 (ICAO 2013).

It includes the dissemination of lessons learned as well as the enhancement of safety culture. In this area as well, practices may vary significantly from one actor to another.

In order to dispatch safety-related information, the air travel industry has progressively launched a number of initiatives. An overall frame has been provided by the ICAO and enforced by local Aviation Authorities but numerous steps in safety communication have been taken by other organizations, resulting in a number of conferences, exchange forums, safety programs and training initiatives. ${ }^{2}$

For a rather long period of time, the major part of safety-related communication took place within the air travel industry itself, including official organizations. It was consistent and to a large extent consensual. The state of air travel safety was clear, as well as the priorities. No external party was interfering and safety was indeed progressing on a continuous basis as proven by statistics. More recently, aviation safety has come increasingly under scrutiny, especially from new stakeholders, not belonging to the traditional circles within this industry.

\section{New Actors, New Safety Communication, New Influences on Safety Management}

Today, other stakeholders, such as victims' associations, law firms, pilot unions, social media, have entered this arena and have earned their share of voice. Through safety-related communication, they claim a right to access all the information and sometimes to perform their own analysis of an event. It is the advent of a system of checks and balances on the historical select club of professionals. Two worlds, two types of practices, one strictly regulated based on a technical approach to safety, the

\footnotetext{
${ }^{2}$ https://flightsafety.org/category/safety-calendar/.
} 
other one 'self-regulated', often driven by reactions to an accident (general public, associations of victims' families such as FENVAC in France, politics, etc.) with different timelines (delayed response due to ongoing investigations vs. immediate response under public pressure).

Aviation accidents have always generated strong media coverage. There are many reasons for this, but we first of all have to recognize that aviation accidents generally result in a significant number of victims. In this respect, they can be considered as big industrial accidents. The overall perception that air travel is an extremely safe means of conveyance also contributes to making each accident a kind of exceptional event. Everybody expects no accident and the safety rate is indeed very high, with one fatal accident every ten million flights for the latest generation of airliners.

When an accident occurs, it contradicts this expectation to a certain extent and the general public, not only aviation professionals and the travelling public, are eager to know what might have happened. This interest in air crashes and subsequent investigations is a well-known fact which materialized in an impressive number of TV reports, films, books and event theatre events.

Back in 1999, a play named Charlie Victor Romeo (CVR also stands for Cockpit Voice Recorder) was created on the basis of audio recordings from several fatal accidents. Actors installed in a cockpit mock-up on a stage would replay the last moments of these crew members. It was a very successful enterprise and Time magazine included Charlie Victor Romeo in their 2004 Best Plays of the Year list. A number of TV series such as 'Air Crash Investigation' produced by Cineflix since 2003 and many others also account for this wide interest in air accidents and the associated investigations. Back in 2012, the famous American actor Denzel Washington played a captain in 'Flight', a successful drama based on a jetliner accident. In 2016, Tom Hanks appeared in the film 'Sully' which related the successful landing of a jetliner in the Hudson River after having hit a number of geese.

Travelling by air is no longer reserved to the wealthy, and millions of people can therefore identify themselves to the unfortunate victims of an airplane accident. There are many reasons for the public at large to be interested in air accidents, but it is a fact and every new unfortunate event confirms this situation. As mentioned previously, accidents are statistically extremely rare but air traffic volume is large and continuously growing. More than 100,000 commercial flights are performed every day, this means more than one aircraft taking off every second.

However, what the public remembers are absolute numbers, not relative ones. And accidents are absolute numbers. It means that safety must progress in proportion as traffic grows if we do not want the general public to develop the feeling that the air transport industry is no longer delivering its promises in terms of safety. Obviously, air accidents coverage always highlights a failure of the safety system at some point. It is clearly negative publicity for the air transport community.

Beyond the general public and its overall interest in these catastrophic events, a number of persons took a far more active share of voice in recent years. It is the case first of all of victims and relatives. The way these communities were treated in the past has not always been to the credit of the air travel industry. Authorities have 
been made aware of the need to clarify everyone's duties and a number of rules have been specified in order to ensure that care, respect and dignity prevail following an accident. The US Federal Family Assistance Plan for Aviation disasters illustrates this approach (NTSB 2008).

Following the accident of the Asiana aircraft in the US, in July 2013, the airline was fined by the Federal Aviation Administration for not providing the expected assistance to families in due time. Victims of an air accident and their families have rights. This is also made more concrete by the level of financial compensation following an aircraft accident, especially in the USA.

Such accidents often generate legal proceedings and some law firms such as Kreindler and Kreindler have specialized in aviation disasters. Because civil damages are substantially higher in the USA, lawyers will often try to sue in this country. The recent accident of Germanwings (March 2015) illustrates this. Some families tried to sue Lufthansa, the parent company of Germanwings, on the basis that the suicidal First Officer was initially trained in the USA. Obviously, law firms will have their own strategies and therefore their own communication objectives following an accident.

Another recent evolution has also seen the growing implication of political staff. On the occasion of the Germanwings accident, three heads of state (President François Hollande from France, Chancellor Angela Merkel from Germany and Prime Minister Mariano Rajoy from Spain) gathered on the crash site for the memorial service. Such a strong presence creates a precedent and is likely to be expected again in comparable cases. This trend can be seen as the reflexion of sociological trends in modern democratic societies. It is nonetheless a confirmation that victims now have a specific and recognized status. This status gives them more influence and their opinions are better heard than before.

Politicians make laws and can change the framework in which safety investigations or justice, for example, are pursued. These are medium to long-term evolutions but they are real possibilities. The family assistance act in the USA is a relevant example of such regulations being introduced. Victims' associations will form quicker than before thanks to the Internet and social media and also because they receive official support from governments. In France, bodies such as INAVEM and FENVAC are officially backed by the authorities in their support to victims and their associations.

Other groups, such as pilot unions, may also get involved in order to protect the reputation and the interests of their members. As a matter of fact, the vast majority of accidents are due to operational factors in which crews often play a part. It is quite common to read articles putting the blame on either the aircraft or the crew. Accident investigations most of the time demonstrate that truth is more complex. Beyond the main cause of an accident, a number of contributing factors may place the event in a different perspective. Understandably, pilots' unions will focus on issues such as Human-Machine Interface in order to explain and defend crew behaviour.

Traditional media will logically follow the move and reflect these numerous activities surrounding an accident whereas social media will generate additional communication involving more actors. The multiplication of $24 \mathrm{~h}$ news channels 
has also increased the coverage of events such as aircraft accidents. The media investigation into the potential causes of the accident will start almost immediately with the support of numerous private experts who quickly elaborate possible scenarios even in the absence of consolidated facts.

It has now become current to see dedicated sites launching their own investigations with limited information from various origins such as flightradar24, weather reports, pictures and videos. One of these groups is Aviation Herald, a site based in Austria and run by Simon Hradecky. It is extremely reactive and has become a kind of reference for several media. Other communities share extensive information quite quickly following an accident or a serious event. ${ }^{3}$

While communication from the aviation community during an accident investigation is highly regulated by the ICAO Annex 13 (ICAO, 2016) which specifies the conditions of an investigation following an accident or a serious incident, the emerging 'new' communication is not subject to such constraints. However, a kind of self-regulation can be observed, especially on social media with common intense exchanges and challenges of untrue statements. This phenomenon by the way leads to the emergence of some influencers recognized by a large community for their expertise. ${ }^{4}$ They enjoy a real status and are taken very seriously by airlines as well as other aviation stakeholders such as Original Equipment Manufacturers.

Eventually, safety communication has progressively increased leading to more information exchange with more actors among aviation professionals, taking place in a continuous manner, silent and invisible to the public. What changed dramatically over the past decade is the safety communication landscape following an accident.

\section{To What Extent Does the Evolution of Safety Communication Impact Safety Management?}

The emergence of new safety communication actors such as victims' associations, justice, political staff, social media has led to an evolution of pressures, stakes and power that eventually influence the way safety is managed. It is worth noting that these new actors' primary motivation is not necessarily to contribute to safety enhancement. Motivations include the right to know, the request to perform an independent analysis, but also an opportunity to promote convictions about technology, training or any other aspect of the aviation industry.

With the strict rules imposed by ICAO Annex 13 on communication about accident investigations (ICAO 2001), requiring an exclusively consolidated factual

\footnotetext{
${ }^{3}$ See for example: http://www.airliners.net/forum/; http://www.pprune.org/.

${ }^{4}$ See for example the following significant influencers on twitter:

Jason Rabinowitz https://twitter.com/AirlineFlyer

Alex Macheras https://twitter.com/AlexInAir

Mary Kirby « Runway Girl » https://twitter.com/RunwayGirl.
} 
basis, the pace of official communication induces a timing that leaves an empty space for too long a time with respect to the current communication and societal landscape. The delay of official communication often creates frustration from media and families and feeds impressions that there is a lack of transparency and that pressure might be applied on authorities in order to protect industrial interests. Such a situation is often observed and may undermine the credibility of investigating authorities.

This lack of trust in investigation bodies also involves an almost systematic challenge of their accident reports. This pressure on investigation authorities may delay the publication of accident reports and sometimes influence the content. Eventually, whatever the motivation of the emerging safety communication actors, their share of voice is consequential on safety management.

First of all, the growing appetite for real-time information encourages speculation to compensate for the lack of facts available immediately after the event. In this respect, it is adding pressure on investigation authorities who generally take more time to communicate their first findings, for they need to rely on validated information.

Every accident leads to the expression of a variety of logics with respect to safety. A rational one turned towards safety enhancement that of the industry and more specifically of the investigation body; an emotional one, that of the victims and families of victims; a rational but more individual one, that of individual experts or critics whose visibility will obviously increase on these occasions.

As previously mentioned, all these logics do not share the exclusive objective to enhance safety. It is therefore almost inevitable to see controversies, polemics, delayed decisions, misperceptions. Moreover, the two latter logics sometimes lead to decisions which do not obviously and directly contribute to safety. For example, following the Sharm El Sheikh accident in which 134 French passengers were killed in January 2004, a parliamentary commission was created in France as a response to public emotion. Forty proposals were made the same year. ${ }^{5}$

Among these 40 proposals, some were by nature doomed to failure whereas others were heavily challenged. Indeed, because air travel safety depends on an international system, it is quite challenging for any country to act independently and efficiently. Therefore, the proposals challenging ICAO recommendations for example about pilots licensing were not realistic. Another proposal was made in order to enforce the exclusive use of English in exchanges between crews and air traffic control in France. It was in fact never enforced because of opposition from some pilots' associations in France. Eventually, despite the publicity made around these 40 proposals, it does not seem that this work had an obvious influence on air travel safety.

Later on, following the crash of the Rio-Paris flight in 2009, a law was proposed in order to create an Aviation Safety Authority ('Haute autorité de la sécurité aérienne'). The objective was to control investigation authorities through a board of nine members. The law was not passed but it illustrates how public emotion and political reaction could impact safety management.

\footnotetext{
${ }^{5}$ http://www.assemblee-nationale.fr/12/rap-info/i1717-t1-05.asp\#P6209_573114.
} 
On the other hand, one can argue that the existence of a counter-power, through emerging safety communication actors, can support safety enhancement by putting the aviation industry under scrutiny and forcing it to do even more. Up to now though, it has not led to the identification of new safety issues.

It seems that the contribution is rather in terms of pressure applied to the overall safety system. One can see this as a recurrent move that will prevent the air travel industry from becoming complacent. Given the extremely low rates of accidents achieved by the industry, the public could fear that air transport actors see themselves as having reached a 'good enough' level in terms of safety. From this point of view, new safety communication actors could be seen as a useful power, a kind of watchdog. However, to keep this role that could contribute to safety enhancement, this counter-power needs to keep an appropriate balance. Indeed, going too much in the direction of negative communication impacts the industry, the authorities and their credibility. It may even give credit to alternative and private accident investigations carried out with less expertise and objectivity. This may result in blurred pictures concerning what needs to be done in order to enhance safety.

Within the framework defined by the ICAO Annex 13, investigations are international task forces involving a number of stakeholders with their own objectives and constraints. Any mishandling would be immediately known.

\section{Conclusion}

Safety communication has been for a very long time a continuous activity, undertaken by professionals within the aviation community. It is still the case to a very large extent when it comes to learning from operations and sharing safety-related information in the absence of accidents. However, the communication landscape changes dramatically in case of an accident. If communication used to translate into aviation professionals providing validated information to journalists who in turn informed the public, the sources of information have become diversified, the nature of information as well, not to mention the motivation of those entering the communication scene. In brief, there is on one hand a permanent exercise carried out by professionals, and on the other hand, ad hoc communication triggered by unfortunate events and led by specific and temporary stakeholders.

The safety strategy, and therefore the associated communication delivered by the air travel industry, is a long-term one, somewhat self-centred and raising potential safety issues in a consensual fashion. It is mostly done by aviation experts representing various domains. The other approach is by nature more critical and often challenges the ability of the air travel community to do an effective job in terms of safety. It is done by very diverse actors and increasingly relayed by social media. The industry has no choice but to face this changing and challenging context. 
Ignoring it would just make things worse. Some key actors have already started to change their communication practices after an accident, especially investigation authorities. They have realized that a lack of communication could impact their credibility; two important investigation bodies, the NTSB in the USA and the BEA in France, have done a lot over the past years. They now give public information much more quickly than before and they do take care of victims' associations in a dedicated way.

For example, depending on the magnitude of the event, US investigation authorities may decide to organize daily press conferences and sometimes to use Twitter in order to inform the general public in an efficient and fast way. It must be noted, however, that such initiatives are not always welcomed by some stakeholders who believe that more time must be taken in order to prevent hasty conclusions. The French Transportation Investigation Board (BEA), for example, ensures that at all times families learn first about their official communications. They also publish a list of ongoing serious incident and accident investigations in an effort to enhance transparency.

Nonetheless, communication practices around safety vary significantly among aviation actors. It is also fair to say that talking about air travel safety between accidents is not easy for two main reasons: first of all, media are more interested in news than in general speeches. Second, talking about safety indirectly means talking about accidents and companies are not always comfortable with this.

The industry nonetheless needs to find a way to be more present in this new world of safety-related communication. Failing to do so would leave an empty seat and limit its role to firefighting. Every safety communication specialist knows that it is difficult to react when you are in a defensive mode, which is almost unavoidable following an accident. A way forward for the industry seems to be to explain its efforts to a wider public with an appropriate approach, not waiting for accidents to occur to communicate on the public scene. Improving its image in terms of transparency can only help.

\section{References}

Graeber, C. (1995). Accident prevention strategy.

Havinga, J., \& Dekker, S. (2014). Just culture: Reporting, the line and accountability. Journal of Aviation Management, 49-57. Published by Singapore Aviation Academy, http://uat-web.saa. com.sg/documents/65465/149063/SAA_Journal_2014.pdf/d06550de-9a92-4632-9746c2f2e $874 \mathrm{c} 556$.

ICAO. (2001). Aircraft accident and incident investigation (9th ed.). ICAO.

ICAO. (2013). Safety management. ICAO.

NTSB. (2008). Federal family assistance plan for aviation disasters. NTSB. 
Open Access This chapter is licensed under the terms of the Creative Commons Attribution 4.0 International License (http://creativecommons.org/licenses/by/4.0/), which permits use, sharing, adaptation, distribution and reproduction in any medium or format, as long as you give appropriate credit to the original author(s) and the source, provide a link to the Creative Commons license and indicate if changes were made.

The images or other third party material in this chapter are included in the chapter's Creative Commons license, unless indicated otherwise in a credit line to the material. If material is not included in the chapter's Creative Commons license and your intended use is not permitted by statutory regulation or exceeds the permitted use, you will need to obtain permission directly from the copyright holder. 